\title{
A COMPLETION FOR A QUASI UNIFORM SPACE
}

\section{RONALD A. STOLTENBERG}

1. Introduction. In a recent publication Pervin and Sieber [2] left open the question of whether or not a quasi uniform space had a completion using their definitions. It is the purpose of this paper to answer this question.

The following definitions are as given by Pervin and Sieber [2].

1.1. Definition. A filter $\mathcal{F}$ in a quasi uniform space $(X, \mathcal{U})$ converges to a point $x \in X$ if and only if for each $U \in \mathcal{U}, U(x) \in \mathcal{F}$.

1.2. Definition. A filter $\mathcal{F}$ in a quasi uniform space $(X, \mathfrak{U})$ is Cauchy if and only for each $U \in \mathcal{U}$ there is $x \in X$ such that $U(x) \in \mathcal{F}$.

1.3. Definition. A quasi uniform space $(X, \mathfrak{u})$ is complete if and only if each Cauchy filter in $X$ converges to a point of $X$.

A function $f$ from a quasi uniform space $(X, \mathcal{u})$ to a quasi uniform space $(Y, v)$ is quasi uniformly continuous relative to $\mathcal{U}$ and $v$ if and only if for each $V \in \mathcal{V}$ the set $\{(x, y):(f(x), f(y)) \in \mathcal{V}\} \in \mathcal{U}$. $f$ is a quasi uniform isomorphism if and only if $f$ is one-to-one onto $Y$ and $f^{-1}$ is quasi uniformly continuous. A quasi uniform space $(X, \mathfrak{u})$ can be embedded in a quasi uniform space $(Y, \mathcal{V})$ when there exists a quasi uniform isomorphism from $(X, V)$ onto a subspace of $(Y, V)$. These definitions are the same as those given in [3].

1.4. Definition. A quasi uniform space $(X, \mathcal{u})$ has a completion if and only if $(X, \mathfrak{u})$ can be embedded in a complete quasi uniform space $\left(X^{*}, u^{*}\right)$ in such a way that $X$ is a dense subset of $X^{*}$.

2. Main results. A quasi pseudometric $d$ on a set $X$ is a function from $X \times X$ into the set of positive real numbers such that for $x, y, z \in X:$ (a) $d(x, x)=0$ and (b) $d(x, y) \leqq d(x, z)+d(z, y)$. The pair $(X, d)$ is called a quasi pseudometric space. Compare Kelly [1]. It is easy to see that a quasi pseudometric space $(X, d)$ is a quasi uniform space and that a filter $\mathcal{F}$ in $(X, d)$ is Cauchy if and only if for each $\epsilon>0$ there exists $x \in X$ such that $S_{\epsilon}(x)=\{y: d(x, y)<\epsilon\} \in F$.

2.1. Theorem. Every quasi pseudometric space $(X, d)$ has a completion $\left(X^{*}, d^{*}\right)$ which is a quasi pseudometric space.

Proof. We may and do assume that $d(x, y) \leqq 1$ for all $x, y \in X$. Let

$$
\bar{X}=\left\{\bar{x}: \bar{x}=\left\{x_{n}: n \in N\right\} \text { is a sequence in } X\right\} \text {. }
$$

\section{Define}

Presented to the Society, January 24, 1967; received by the editors July 20, 1966. 


$$
\bar{d}: \bar{X} \times \bar{X} \rightarrow R:\left\{\begin{array}{l}
\bar{d}(\bar{x}, \bar{y})=0 \text { if } \bar{y} \text { is a subsequence of } \bar{x} \\
\bar{d}(\bar{x}, \bar{y})=\lim _{n} \inf \lim _{m} d\left(x_{n}, y_{m}\right) \text { otherwise. }
\end{array}\right.
$$

(a) Lemma. Suppose $\bar{y}$ is not a subsequence of $\bar{x}$. If $\bar{d}(\bar{x}, \bar{y})<\delta$ then for each $K>0$ there exists $k_{0} \geqq K$ and $L\left(k_{0}\right)$ such that for $j \geqq L\left(k_{0}\right)$, $d\left(x_{k_{0}}, y_{j}\right)<\delta$. On the other hand if for each $K>0$ there is $k_{0} \geqq K$ and $L\left(k_{0}\right)$ such that $d\left(x_{k_{0}}, y_{j}\right)<\delta$ whenever $j \geqq L\left(k_{0}\right)$ then $\bar{d}(\bar{x}, \bar{y}) \leqq \delta$.

Proof. Suppose $\bar{d}(\bar{x}, \bar{y})<\delta$. Now $\bar{d}(\bar{x}, \bar{y})=\lim \inf _{n} \lim \sup _{m}$ $d\left(x_{n}, y_{m}\right)=U$. Now $U<\delta$ implies for every $K<0$ there exists $k_{0} \geqq K$ such that $\lim \sup _{m} d\left(x_{k_{0}}, y_{m}\right)=U^{\prime}<\delta . U^{\prime}<\delta$ implies there exists $L\left(k_{0}\right)$ such that for $j \geqq L\left(k_{0}\right), d\left(x_{k_{0}}, y_{j}\right)<\delta$. So we have the first part of the lemma.

On the other hand suppose for each $K>0$ there exists $k_{0} \geqq K$ and $L\left(k_{0}\right)$ such that for $j \geqq L\left(k_{0}\right), d\left(x_{k_{0}}, y_{j}\right)>\delta$. Hence $\lim \sup _{m} d\left(x_{k 0}, y_{m}\right)$ $\leqq \delta$ since for $j \geqq L\left(k_{0}\right), d\left(x_{k_{0}}, y_{j}\right)<\delta$. But now inf $k_{\geq \geq n} \lim \sup _{m} d\left(x_{k}, y_{m}\right) \leqq \delta$ for any $k$ so $\sup _{n} \inf _{k \geqq n} \lim \sup _{m} d\left(x_{k}, y_{m}\right)=\lim \inf _{n} \lim \sup _{m} d\left(x_{n}, y_{m}\right)$ $\leqq \delta$.

(b) Lemma. $\bar{d}$ is a quasi pseudometric for $\bar{X}$.

Proof. Clearly $\bar{d}(\bar{x}, \bar{x})=0$ for all $\bar{x} \in \bar{X}$. Let $\bar{x}, \bar{y}, \bar{z} \in \bar{X}$. To show that $\bar{d}(\bar{x}, \bar{y}) \leqq \bar{d}(\bar{x}, \bar{z})+\bar{d}(\bar{z}, \bar{y})$ there are four cases to consider:

Case 1. $\bar{y}$ is a subsequence of $\bar{x}$.

In this case $\bar{d}(\bar{x}, \bar{y})=0 \leqq \bar{d}(\bar{x}, \bar{z})+\bar{d}(\bar{z}, \bar{y})$.

Case 2. $\bar{z}$ is a subsequence of $\bar{x}$ and $\bar{y}$ not a subsequence of $\bar{x}$.

In this case $\bar{d}(\bar{x}, \bar{z})=0$. Suppose $\bar{d}(\bar{z}, \bar{y})<\bar{d}(\bar{x}, \bar{y})$. Let $\delta=\bar{d}(\bar{x}, \bar{y})$ $-\bar{d}(\bar{z}, \bar{y})$ now $\bar{d}(\bar{z}, \bar{y})<\bar{d}(\bar{z}, \bar{y})+\delta / 2$. So for each $P>0$ there is $p_{0} \geqq P$ and $L\left(p_{0}\right)$ such that $d\left(z_{p_{0}}, y_{j}\right)<\bar{d}(\bar{z}, \bar{y})+\delta / 2$ whenever $j \geqq L\left(p_{0}\right)$. However $\bar{z}$ is a subsequence of $\bar{x}$. So $z_{p_{0}}=x_{n_{p_{0}}}$ where $n_{p_{0}} \geqq p_{0} \geqq P$. So for each $P>0$ there is $n_{p_{0}} \geqq P$ and $L\left(n_{p_{0}}\right)=L\left(p_{0}\right)$ such that $d\left(x_{x_{n p}}, y_{j}\right)$ $<\bar{d}(\bar{z}, \bar{y})+\delta / 2$ for all $j \geqq L\left(n_{p_{0}}\right)$. So by Lemma (a) $\bar{d}(\bar{x}, \bar{y}) \leqq \bar{d}(\bar{z}, \bar{y})$ $+\delta / 2<\bar{d}(\bar{x}, \bar{y})$, a contradiction. So $\bar{d}(\bar{x}, \bar{y}) \leqq \bar{d}(\bar{x}, \bar{z})+\bar{d}(\bar{z}, \bar{y})$.

Case 3. $\bar{y}$ is a subsequence of $\bar{z}$ and $\bar{y}$ is not a subsequence of $\bar{x}$. In this case $\bar{d}(\bar{z}, \bar{y})=0$. Suppose $\bar{d}(\bar{x}, \bar{z})<\bar{d}(\bar{x}, \bar{y})$. Let $\delta=\bar{d}(\bar{x}, \bar{y})$ $-\bar{d}(\bar{x}, \bar{z})$. Now $\bar{d}(\bar{x}, \bar{z})<\bar{d}(\bar{x}, \bar{z})+\delta / 2$. So for each $P>0$ there is $p_{0} \geqq P$ and $L\left(p_{0}\right)$ such that $d\left(x_{p_{0}}, z_{j}\right)<\bar{d}(\bar{x}, \bar{z})+\delta / 2$ for all $j \geqq L\left(p_{0}\right)$. However $\bar{y}$ is a subsequence of $\bar{z}$. So $y_{k}=z_{j_{k}}$. Now for $j_{k} \geqq L\left(p_{0}\right)$, $d\left(x_{p_{0}}, z_{j_{k}}\right)=d\left(x_{p_{0}}, y_{k}\right)<\bar{d}(\bar{x}, \bar{z})+\delta / 2$. Let $L^{\prime}\left(p_{0}\right)$ be the smallest integer such that $j_{L\left(p_{0}\right)} \geqq L\left(p_{0}\right)$. Thus for each $P>0$ there is $p_{0} \geqq P$ and $L^{\prime}\left(p_{0}\right)$ such that $d\left(x_{p_{0}}, y_{k}\right)<\bar{d}(\bar{x}, \bar{z})+\delta / 2$ for all $k \geqq L^{\prime}\left(p_{0}\right)$. So $\bar{d}(\bar{x}, \bar{y}) \leqq \bar{d}(\bar{x}, \bar{z})$ $+\delta / 2<\bar{d}(\bar{x}, \bar{y})$, a contradiction. So $\bar{d}(\bar{x}, \bar{y}) \leqq \bar{d}(\bar{x}, \bar{z})+\bar{d}(\bar{z}, \bar{y})$. 
Case 4. $\bar{z}$ is not a subsequence of $\bar{x}, \bar{y}$ is not a subsequence of $\bar{z}$ and $\bar{y}$ is not a subsequence of $\bar{x}$.

In this case $\bar{d}(\bar{x}, \bar{y})=\lim \inf _{n} \lim \sup _{m} d\left(x_{n}, y_{m}\right), \bar{d}(\bar{x}, \bar{z})=\lim \inf _{n}$ $\lim \sup _{m} d\left(x_{n}, z_{m}\right)$ and $\bar{d}(\bar{z}, \bar{y})=\lim \inf _{n} \lim \sup _{m} d\left(z_{n}, y_{m}\right)$. Let $\delta>0$; let $U_{1}=\bar{d}(\bar{x}, \bar{z})$; let $U_{2}=\bar{d}(\bar{z}, \bar{y})$. Let $K>0$.

(1) $U_{1}<U_{1}+\delta / 2$ implies there exists $k_{0} \geqq K$ and $L\left(k_{0}\right)$ such that for $j \geqq L\left(k_{0}\right), d\left(x_{k_{0}}, z_{j}\right)<U_{1}+\delta / 2$.

(2) $U_{2}<U_{2}+\delta / 2$ implies there exists $j_{0} \geqq L\left(k_{0}\right)$ and $L\left(j_{0}\right)$ such that for $s \geqq L\left(j_{0}\right), d\left(z_{j_{0}}, y_{s}\right)<U_{2}+\delta / 2$.

Now $d\left(x_{k_{0}}, y_{s}\right) \leqq d\left(x_{k_{0}}, z_{j_{0}}\right)+d\left(z_{j_{0}}, y_{s}\right)<U_{1}+U_{2}+\delta$. So for each $K>0$ there exists $k_{0} \geqq K$ and $L^{\prime}\left(k_{0}\right)=L\left(j_{0}\right)$ such that for $s \geqq L^{\prime}\left(k_{0}\right)$, $d\left(x_{s}, y_{k_{0}}\right)<U_{1}+U_{2}+\delta$. Hence by Lemma (a) $\bar{d}(\bar{x}, \bar{y}) \leqq U_{1}+U_{2}$ $=\bar{d}(\bar{x}, \bar{z})+\bar{d}(\bar{z}, \bar{y})$.

It follows that $\bar{d}$ is a quasi pseudometric on $X$.

(c) Lemma. $(\bar{X}, \bar{d})$ is a complete quasi pseudometric space.

Proof. Let $\mathcal{F}$ be a Cauchy filter in $(\bar{X}, \bar{d})$. Then for each integer $k$ there is $\bar{x}^{k} \in \bar{X}$ such that $S_{k}\left(\bar{x}^{k}\right)=\left\{\bar{y}: \bar{d}\left(\bar{x}^{k}, \bar{y}\right)<\frac{1}{2} k\right\} \in \mathcal{F}$. Now $\bar{x}^{k}$ $=\left\{x_{j}^{k}: j \in N\right\}$ for each $k$. Let $\bar{x}=\left\{x_{1}^{1}, x_{1}^{2}, x_{2}^{1}, x_{1}^{3}, x_{2}^{2}, x_{3}^{1}, \cdots\right.$, $\left.x_{1}^{k}, x_{2}^{k-1}, x_{3}^{k-2}, \cdots, x_{k}^{1}, \cdots\right\}=\left\{\left\{x_{m(j, h-j)}: j=1,2, \cdots, h-1\right\}:\right.$ $h=2,3, \cdots\}$ where $x_{m(j, h-j)}=x_{j}^{h-j}$ and $m(j, h-j)=((h-2)(h-1)) / 2$ $+j$. Clearly $\bar{x}^{k}$ is a subsequence of $\bar{x}$ for each $k$. So $\bar{d}(\bar{x}, \bar{y}) \leqq \bar{d}\left(\bar{x}, \bar{x}^{k}\right)$ $+\bar{d}\left(\bar{x}^{k}, \bar{y}\right)<\frac{1}{2}{ }^{k}$ whenever $\bar{y} \in S_{k}\left(\bar{x}^{k}\right)$. So $S_{k}\left(\bar{x}^{k}\right) \subseteq S_{k}(\bar{x})$. Hence $S_{k}(\bar{x}) \in \mathcal{F}$ for each $k$. Thus $\mathcal{F}$ converges to $\bar{x}$ in $\bar{X}$. So $(\bar{X}, \bar{d})$ is complete.

(d) Lemma. $(X, d)$ can be embedded in $(\bar{X}, \bar{d})$.

Proof. Let $\phi:(X, d) \rightarrow(\bar{X}, \bar{d}): \phi(x)=\left\{x_{n}: n \in N\right\}$ where $x_{n}=x$ for all $n \in N . \bar{d}(\phi(x), \phi(y))=\lim \inf _{n} \lim \sup _{m} d\left(x_{n}, y_{m}\right)=d(x, y)$; thus it follows that $\phi$ is a quasi uniform isomorphism from $(X, d)$ onto a subspace denoted by $X$ of $(\bar{X}, \bar{d})$. Hence $(X, d)$ can be embedded in $(\bar{X}, \bar{d})$.

Let $X^{*}$ be the closure of $X$ in $(\bar{X}, \bar{d})$ and let $d^{*}=\bar{d} \mid \bar{X}$. Clearly $\left(X^{*}, d^{*}\right)$ is a completion for $(X, d)$. This completes the proof of 2.1 .

2.2. Proposition. A closed subspace of a complete quasi uniform space is complete.

2.3. Proposition. Let $\left(X_{\alpha}^{*}, \boldsymbol{u}_{\alpha}\right)$ be a quasi uniform space for each $\alpha$ in an index set $\Omega$. Let $X_{\alpha \in \Omega}\left\{X_{\alpha}\right\}=X$ and let the product quasi uniformity be $\mathfrak{u}$. Then $(X, \mathfrak{u})$ is complete if and only if $\left(X_{\alpha}, \mathfrak{u}_{a}\right)$ is complete for each $\alpha \in \Omega$.

The proofs of Propositions 2.2 and 2.3 are clear and are omitted. 
2.4. Theorem. Every quasi uniform space $(X, \mathfrak{u})$ has a completion $\left(X^{*}, u^{*}\right)$.

Proof. By a theorem in [3] we have that $(X, \mathfrak{u})$ can be embedded in a product of quasi pseudometric space $X\left\{\left(X_{\alpha}, d_{\alpha}\right): \alpha \in \Omega\right\}$. We may and do assume $d_{\alpha}\left(x_{\alpha}, y_{\alpha}\right) \leqq 1$ for all $x_{\alpha}, y_{\alpha} \in X_{\alpha}$ and for each $\alpha \in \Omega$. Each space $\left(X_{\alpha}, d_{\alpha}\right)$ has a completion $\left(X_{\alpha}^{*}, d_{\alpha}^{*}\right)$. Clearly $(X, \mathfrak{u})$ can be embedded in $X\left\{\left(X_{\alpha}^{*}, d_{\alpha}\right): \alpha \in \Omega\right\}$. Let $X^{*}$ be the closure of $X$ in $X\left\{\left(X_{\alpha}^{*}, d_{\alpha}^{*}\right): \alpha \in \Omega\right\}$. Let $u^{*}=\left\{U \cap X^{*} \times X^{*}: U\right.$ is a member of the product quasi uniformity for $\left.X\left\{X_{\alpha}^{*}: \alpha \in \Omega\right\}\right\}$. From Propositions 2.2 and 2.3 it follows that $\left(X^{*}, \mathfrak{u}^{*}\right)$ is a completion for $(X, \mathfrak{u})$.

\section{REFERENCES}

1. J. C. Kelly, Bitopological spaces, Proc. London Math Soc. 13 (1963), 71-89.

2. J. L. Sieber and W. J. Pervin, Completeness in quasi-uniform spaces, Math Ann., 158 (1965), 79-81.

3. R. A. Stoltenberg, Some properties of quasi-uniform spaces, Proc. London Math. Soc. 17 (1967), 226-240.

Washington State University 